\title{
NUEVOS RESTOS TERCIARIOS DE SPHENISCIDAE (AVES, SPHENISCIFORMES) PROCEDENTES DE LA COSTA DEL PERÚ
}

\author{
Carolina ACOSTA HOSPITALECHE ${ }^{1}$ y Marcelo \\ STUCCHI \\ ${ }^{1}$ CONICET Departamento Científico Paleontología Vertebrados. Museo de La \\ Plata. Paseo del Bosque s/n, 1900. La Plata. Argentina. \\ acostacaro@museo.fcnym.unlp.edu.ar \\ ${ }^{2}$ Asociación Ucumari. Jr. Los Agrólogos 220. Lima 12. Perú. \\ asociacion_ucumari@yahoo.com
}

Acosta Hospitaleche, C. \& Stucchi, M. 2005. Nuevos restos terciarios de Spheniscidae (Aves, Sphenisciformes) procedentes de la costa del Perú. [New Tertiary remains of Spheniscidae (Aves, Sphenisciformes) of the coast of Peru.] Revista Española de Paleontología, 20 (1), 1-5. ISSN 0213-6937.

\begin{abstract}
Two new remains of Spheniscidae from the coast of Perú are studied. One of them, a tarsometatarsus, from the Zamaca Locality, Department of Ica, was exhumed of sediments of the Chilcatay Formation (early Middle Miocene) and it is assigned to Palaeospheniscus sp. This finding constitutes the first mention of Spheniscidae in the Chilcatay Formation, being it the most northern record for the Subfamily Palaeospheniscinae. It allows extending its distribution area to the Middle Miocene of the coast of Perú. The second one, belonging to a tibiotarsus from the Bajada del Diablo Locality, Department of Ica, was collected in the Otuma Formation (Late Eocene - Early Oligocene) and assigned to a Spheniscidae indet. which corresponds to a different species of that one of the tarsometatarsus.
\end{abstract}

Key words: Spheniscidae, Palaeospheniscus, early Middle Miocene, Chilcatay Formation, Late EoceneEarly Oligocene, Otuma Formation, Perú.

\section{RESUMEN}

Se estudian dos nuevos restos de Spheniscidae procedentes de la costa peruana. Uno de ellos, un tarsometatarso, proveniente de la Localidad de Zamaca, Departamento de Ica, fue exhumado de sedimentos de la Formación Chilcatay (Mioceno Medio temprano) y es asignado a Palaeospheniscus sp. Este nuevo hallazgo constituye la primera cita para la Formación Chilcatay, constituyendo el registro más septentrional conocido para la Subfamilia Palaeospheniscinae. Esto permite ampliar su rango de distribución extendiéndolo al Mioceno Medio de la costa del Perú. El segundo, correspondiente a un tibiotarso, de la Localidad de Bajada del Diablo, Departamento de Ica, fue colectado en la Formación Otuma (Eoceno Tardío - Oligoceno Temprano) y es asignado a un Spheniscidae indet., siendo posible agregar que pertenece a un taxón distinto a aquel del tarsometatarso.

Palabras clave: Spheniscidae, Palaeospheniscus, Mioceno Medio temprano, Formación Chilcatay, Eoceno Tardío- Oligoceno Temprano, Formación Otuma, Perú.

\section{INTRODUCCIÓN}

Los Spheniscidae constituyen un grupo de aves exclusivamente marinas y de hábitos buceadores. Su distribución se restringe a los mares australes y su presencia en bajas latitudes se encuentra asociada con corrientes marinas de aguas frías (Martínez, 1992).
Se conocen seis géneros de pingüinos actuales, que reúnen un total de 17 especies, en la Subfamilia Spheniscinae (Simpson, 1946), nueve de las cuales habitan el cono sur de América, distribuyéndose en los mares y las costas de $\mathrm{Ar}$ gentina, Chile, Perú hasta la Antártida (Martínez, 1992).

Los representantes fósiles del orden se clasifican en cuatro subfamilias, Paraptenodytinae, Palaeospheniscinae, 
Anthropornithinae y Palaeeudyptinae (Simpson, 1946), además de una probable nueva subfamilia solamente conocida para el Mioceno Tardío temprano de Patagonia (Acosta Hospitaleche, 2004). Las tres primeras, están representadas en América del Sur, en localidades que se encuentran dentro o cerca del área de distribución actual del orden.

Para América del Sur se conocen al menos once especies procedentes de la Patagonia, Argentina (Simpson, 1972, 1981; Cione \& Tonni, 1981; Cozzuol et al., 1993), cinco para Chile (Walsh \& Hume, 2001; Acosta Hospitaleche et al., 2002) y dos para Perú (Stucchi, 2002; Stucchi et al., 2003). Específicamente en Perú, el estudio de los Spheniscidae fue iniciado por Hoffstetter (1968) y posteriormente retomado por Muizon \& DeVries (1985) quienes mencionan la probable presencia de dos nuevos taxones de Spheniscidae para la Formación Pisco (Mioceno Tardío - Plioceno Temprano): uno en el área de Sacaco y el otro, de menor talla, en el área de Ocucaje. En una nueva revisión, Marocco \& Muizon (1988) asignan preliminarmente estos materiales a un Spheniscidae indet. distinto de Spheniscus Brisson, 1760. Posteriomente, Noriega \& Tambussi (1989), señalan el hallazgo de un tarsometatarso de gran tamaño procedente del Mioceno Tardío de la Formación Pisco que sería asignable a un nuevo género y especie de Spheniscinae afín a Eudyptes Vieillot, 1816 y Spheniscus. Recientemente, han sido descriptas dos nuevas especies del género actual Spheniscus (Stucchi, 2002; Stucchi et al., 2003), procedentes de varios niveles de la Formación Pisco. La primera de ellas, S. urbinai Stucchi, 2002 está fundada en un esqueleto parcialmente articulado (Stucchi, 2002), mientras que la segunda, S. megaramphus Stucchi, Urbina \& Giraldo, 2003, en varios elementos craneales (Stucchi et al., 2003).

En el presente trabajo, son estudiados dos nuevos restos, recientemente colectados, que proceden de la costa de Perú.

\section{MATERIAL Y MÉTODOS}

Los restos fósiles fueron descriptos siguiendo la terminología de Baumel \& Witmer (1993) y las medidas fueron tomadas con un calibre Vernier. Se consultaron las colecciones del Museo de Ciencias Naturales "Bernardino Rivadavia", de la Ciudad Autónoma de Buenos Aires (MACN) y Museo de La Plata, de la Ciudad de La Plata (MLP), Argentina; Museo Nacional de Historia Natural (MNHN) de la ciudad de Santiago, Chile; Museo de Historia Natural de la Universidad de San Marcos, Departamento de Paleontología de Vertebrados (MUSM) de Lima, Perú; Field Museum of Natural History (FMNH) de Chicago y Museo de la Universidad de Florida (UF) de Gainesville, Estados Unidos. Los ejemplares están depositados en el Museo de Historia Natural de la Universidad de San Marcos (MUSM) de Lima, Perú.

\section{PALEONTOLOGÍA SISTEMÁTICA}

\section{ORDEN SPHENISCIFORMES Sharpe, 1891}

Familia Spheniscidae Bonaparte, 1831

Subfamilia Palaeospheniscinae Simspon, 1946

Género Palaeospheniscus Moreno \& Mercerat, 1891

Palaeospheniscus sp.

Fig. 1

Material: MUSM 257, tarsometatarso derecho.

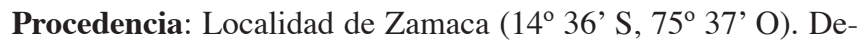
partamento de Ica, costa centro-sur del Perú (Fig. 2). Formación Chilcatay. Esta unidad, según datos éditos, correspondería al Oligoceno Tardío - Mioceno Temprano (De Vries, 1998), pero recientes investigaciones permitirían asignar su parte superior al Mioceno Medio temprano (De Vries, com. pers).

Medidas (expresadas en mm): Longitud total: 38,0; ancho proximal: 16,3; ancho mínimo de la diáfisis: 15,5; ancho distal: 18,4; ancho de la trochlea para el dígito III: 6,5 .

Comentarios: La Subfamilia Palaeospheniscinae se diferencia de los Paraptenodytinae, Anthropornithidae y Spheniscinae por poseer el foramen vasculare proximale mediale rudimentario en comparación con el foramen vasculare proximale laterale y de los Palaeeudyptinae por la presencia de ambas foramina vascularia proximalis. Adicionalmente, su gran elongación lo diferencia de los

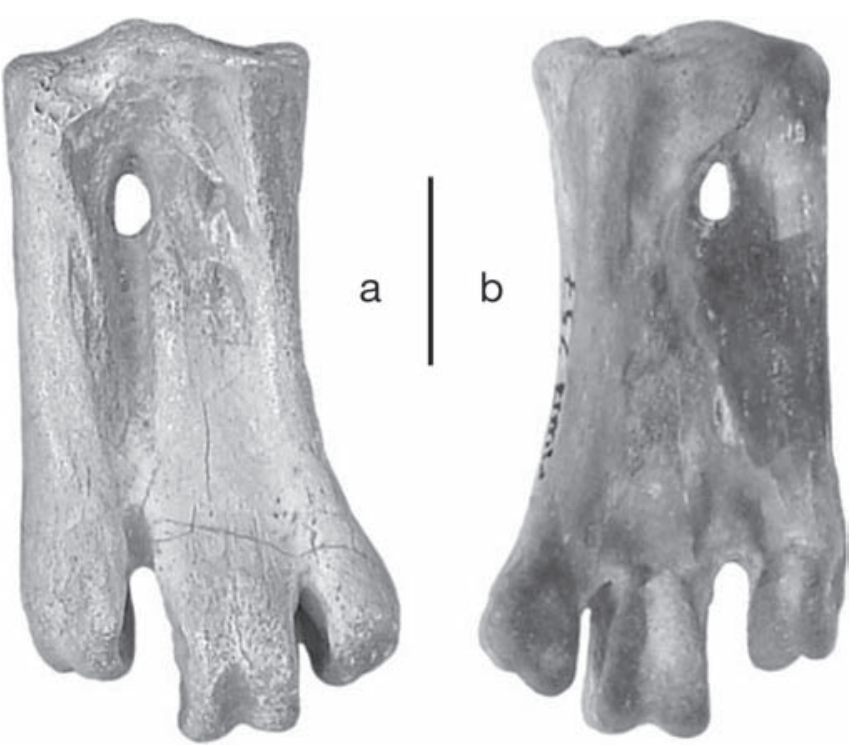

Figura 1. MUSM 257 Palaeospheniscus sp., tarsometatarso derecho. a. vista craneal; b. vista caudal. Escala: 10 $\mathrm{mm}$.

MUSM 257 Palaeospheniscus sp., right tarsometatarsus. a. cranial view; b. caudal view. Scale bar: 10 $m m$. 


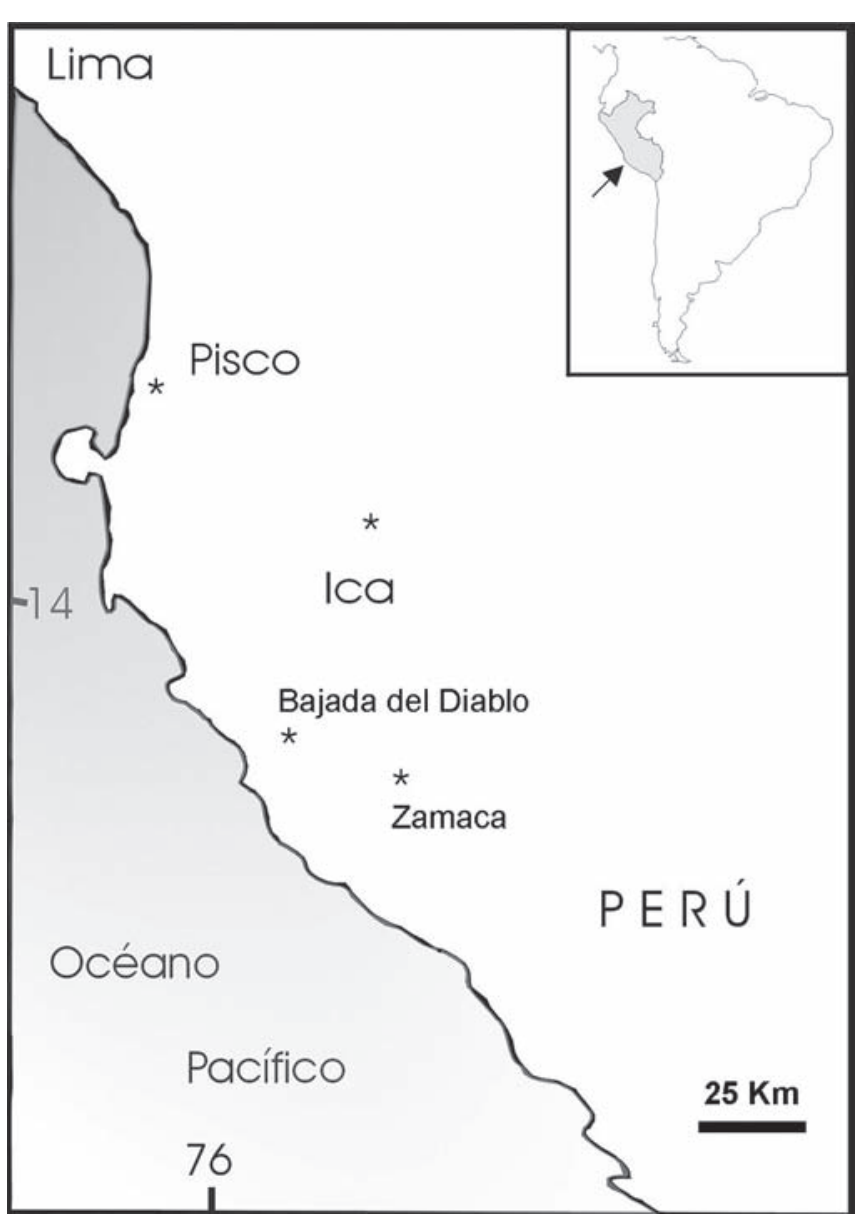

Figura 2. Mapa de ubicación. Location map.

Palaeeudyptinae, Paraptenodytinae y Spheniscinae, mientras que la fuerte fusión de los metatarsales lo distingue de los Spheniscinae. Consecuentemente, la combinación de los siguientes caracteres permite asignarlo a la Subfamilia Palaeospheniscinae: forma alargada con un índice de elongación (longitud máxima/ ancho proximal) mayor que 2, sulcus longitudinalis dorsalis lateralis y medialis menos profundos que en Spheniscus, especialmente el medialis que no se prolonga más allá de la mitad de la facies cranialis, incisuras intertrochlearis medialis y lateralis alcanzando el mismo nivel que en Spheniscus, mientras que en Palaeospheniscus, esta última se prolonga hasta un nivel más proximal, foramen vasculare proximale bien desarrollado y foramen vasculare proximale mediale muy reducido y no abierto sobre la facies caudalis, fossa infracotylaris dorsalis medialis triangular, crista lateralis hypotarsi menos desarrollada que la crista medialis hypotarsi.

Los Palaeospheniscinae (Tabla 1), según Acosta Hospitaleche (2004), se encuentran representados por las siguientes especies: Eretiscus tonni (Simpson, 1981), Palaeospheniscus bergi Moreno \& Mercerat, 1891, P. patagonicusi Moreno \& Mercerat, 1891 y P. biloculata (Simpson, 1970).
La asignación a Palaeospheniscus resulta posible debido a la presencia de los siguientes caracteres: fossa supratrochlearis plantaris extendida proximalmente a las trócleas II y III, disminuyendo su profundidad hacia la tróclea IV, abarcando un área considerablemente mayor a la que ocupa en Spheniscus, foramen vasculare proximale mediale no visible en vista caudal y foramen vasculare proximale laterale abierto lateral y externamente a las crestas del hipotarso, lateral externo conformado por una lámina ósea que proyecta un filoso reborde dirigido hacia la facie caudalis, que no se encuentra presente en Spheniscus.

Las tres especies del género Palaeospheniscus se distinguen principalmente por su talla y resultan morfológicamente indiferenciables entre sí. De acuerdo a estos criterios, el MUSM 257 puede ser asignado solamente a nivel genérico.

\section{Familia Spheniscidae indet.}

Fig. 3.

Material: MUSM 224, tibiotarso izquierdo.

Procedencia: Localidad de Bajada del Diablo (14 ${ }^{\circ} 27^{\prime} \mathrm{S}$, 75 56' O). Departamento de Ica, costa central del Perú. Formación Otuma (Fig. 2) Los datos publicados asignan esta unidad al Oligoceno inferior (DeVries, 1998), aunque recientes estudios permitirían pensar que estos estratos serían aún más antiguos, situándolos en el Eoceno Tardío - Oligoceno Temprano, ca. 36 Ma. (DeVries, 2004).

Medidas (expresadas en mm): Longitud total: 207,4; ancho proximal: 26,8; ancho distal: 30,2.

Comentarios: Aunque el estado fragmentario de este material no permite realizar una asignación sistemática más precisa, fue posible comparar los siguientes caracteres. El tuberculum retinaculi musculi fibularis es más conspicuo que en Spheniscus magellanicus (Forster, 1781), la

\begin{tabular}{lccc}
\hline \multicolumn{1}{c}{ Especie } & $\mathrm{L}$ & $\mathrm{AP}$ & $\mathrm{AD}$ \\
\hline E. tonni & ca.20 & ca.8 & - \\
P. bergi & $30-36$ & $14-16$ & $16-20$ \\
P. patagonicus & $38-40$ & $15-18$ & $18-21$ \\
P. biloculata & 43 & $19-20$ & $19-22$ \\
\hline
\end{tabular}

Tabla 1. Medidas de los tarsometatarsos de las especies de Palaeospheniscinae ( $c f$. Acosta Hospitaleche, 2004), expresadas en milímetros. L: longitud máxima; AP: ancho proximal; AD: ancho distal.

Measurements of the tarsometatarsus of the species of Palaeospheniscinae (cf. Acosta Hospitaleche, 2004), in milimetres. L: maximum length; AP: proximal width; AD: distal width. 

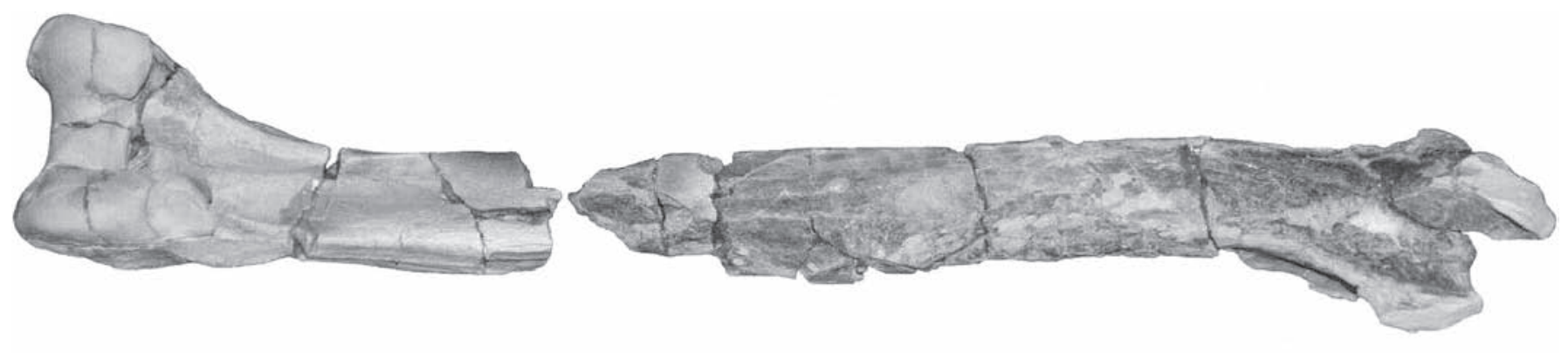

Figura 3. MUSM 224, Spheniscidae indet. tibiotarso izquierdo. MUSM 224, Spheniscidae indet. left tibiotarsus.

tuberositas popliteus es ancha y se encuentra dividida en dos por un surco, la facies articularis medialis es menor que la lateralis, mientras que esta relación es inversa en S. humboldti Meyen, 1834. Sobre el condylus lateralis se presenta una fosa que no se observa en $S$. humboldti. También aparece una ligera depresión sobre el epicondylus medialis. La distancia anteroposterior a nivel distal es ligeramente mayor que el ancho latero-medial. El sulcus intercnemialis se extiende hasta el extremo proximal conservando la misma profundidad en todo su recorrido como en Paraptenodytes antarctica. El pons supratendinosus es ancho y su superficie presenta una relación ligeramente oblicua respecto a la facies cranialis. Sobre el lateral externo de este puente, se presenta un tubérculo proporcionalmente más robusto que en Spheniscus. El canalis extensorius posee sección oval. Sobre el reborde interno del sulcus extensorius se presenta un tubérculo poco marcado. La fossa flexoria es más superficial que en Spheniscus. El epicondylus medialis se proyecta formando una única prominencia redondeada.

\section{DISCUSIÓN}

La Subfamilia Palaeospheniscinae únicamente se encuentra registrada hasta el momento en América del Sur. El género Paleospheniscus ha sido citado para la Formación Bahía Inglesa (Mioceno Superior- Plioceno Inferior) de Chile (Acosta Hospitaleche et al., 2002.) y la Formación Gaiman (Mioceno Inferior) de Argentina (Simpson, 1946, 1972). Este nuevo hallazgo constituye la primera cita de un pingüino para la Formación Chilcatay y el registro más septentrional conocido para la Subfamilia Palaeospheniscinae. Esto permite ampliar su rango de distribución extendiéndolo hasta la costa peruana durante el Mioceno Medio, de acuerdo a los datos estratigráficos mencionados. Además, este resto constituye el segundo vertebrado después de Sula sp. (Stucchi \& DeVries, 2003) reportado para esta unidad.
Simpson (1946) al definir las subfamilias de Spheniscidae basó su diagnosis en las características de los tarsometatarsos y de los húmeros, por lo que no ha sido posible, en este caso, conocer en qué subfamilia se podría incluir el tibiotarso estudiado. De todos modos, su tamaño es sustancialmente mayor que el del tarsometatarso MUSM 257 lo que permite descartar que ambos restos pertenezcan al mismo taxón.

\section{AGRADECIMIENTOS}

A Mario Urbina y Vinnie Valle por colectar los materiales, sus valiosos comentarios y permitirnos el estudio de los mismos. A Claudia Tambussi por la lectura crítica de una versión preliminar de este manuscrito. MS desea también agradecer a Rodolfo Salas (Museo de Historia Natural de San Marcos), John Bates, Paul Velazco, José Tello y David Willard (Field Museum of Natural History), Richard Hulbert, Bruce MacFadden y David Steadman (Universidad de Florida), por permitirle revisar las colecciones de sus museos y las facilidades brindadas.

\section{BIBLIOGRAFÍA}

Acosta Hospitaleche, C. 2004. Los pingüinos (Aves, Sphenisciformes) fósiles de Argentina. Sistemática, biogeografía y evolución. Tesis doctoral, Facultad de Ciencias Naturales y Museo, UNLP, 321 pp. (inédita).

Acosta Hospitaleche, C., Fritis, O., Tambussi, C. \& Quinzio, A. 2002. Nuevos restos de pingüinos (Aves: Spheniscidae) en la Formación Bahía Inglesa (Mioceno superior - Plioceno inferior) de Chile. $1^{\circ}$ Congreso Latinoamericano de Paleontología de Vertebrados, Santiago de Chile, 16.

Ameghino, F. 1905. Enumeración de los impennes fósiles de Patagonia y de la isla Seymour. Anales del Museo Nacional de Buenos Aires, 3, 97-167.

Baumel, J. \& Witmer, L.M. 1993. Osteologia. In: Handbook of avian anatomy: Nomina Anatomica Avium (Coord. J. Baumel, A. King, J. E. Breazile, H. Evans \& J. C. Vanden Bergue). Publications of the Nuttall Ornithological Club, 
Harvard University, Cambridge, Massachusetts, 45-132.

Cione, A. \& Tonni, E. 1981. Un pingüino de la Formación Puerto Madryn (Mioceno Tardío) de Chubut, Argentina. Comentarios acerca del origen, la paleoecología y zoogeografía de los Spheniscidae. Anais II Congresso LatinoAmericano Paleontología, Porto Alegre, 2, 591-604.

Cozzuol, M. A., Tambussi, C. \& Noriega, J. 1993. Un pingüino (Aves: Spheniscidae) de la Formación Puerto Madryn (Mioceno Medio) en Península Valdés, Chubut, Argentina, con importantes implicancias filogenéticas. Ameghiniana, 30, 327-328.

DeVries, T. J. 1998. Oligocene deposition and Cenozoic sequence boundaries in the Pisco Basin, Perú. Journal of South American Earth Sciences, 11, 217-231.

DeVries, T. J. 2004. Eocene mollusks from the Pisco Basin (Southern Peru): Evidence for re-evaluating the age of the Otuma Formation. XII Congreso Peruano de Geología, Lima, Octubre 2004. Resúmenes extendidos, 436-439.

Hoffstetter, M. R. 1968. Un gisement de vertébrés tertiaires à Sacaco (Sud-Pérou), témoin néogene d'une migration de faunes australes au long de la côte occidentale sudaméricaine. Comptes Rendus de l'Académie des Science de Paris, 267, 1273-1276.

Marocco, R. \& Muizon, C. de. 1988. Los Vertebrados del Neógeno de la costa Sur del Perú. Boletín del Instituto Francés de Estudios Andinos, 17, 105-117.

Martínez, I. 1992. Order Sphenisciformes. In: Handbook of the birds of the world, Vol 1 Ostrich to Ducks (Coord. J. del Hoyo, A. Elliott \& J. Sargatal). Lynx Editions, Barcelona, 140-160.

Moreno, F. P. \& Mercerat, A. 1891. Catálogo de los pájaros fósiles de la República Argentina conservados en el Museo de La Plata. Anales del Museo de La Plata (Paleontología Argentina), 1, 7-71.

Muizon, C. de \& DeVries, T. 1985. Geology and paleontology of late Cenozoic marine deposits in the Sacaco area (Perú). Geolische Rundschau, 74, 547-563.

Noriega, J. \& Tambussi, C. 1989. Un Spheniscidae (Aves: Sphenisciformes) del Mioceno Tardío de la costa del Perú. Ameghiniana, 26, 247.

Simpson. G. G. 1946. Fossil Penguins. Bulletin Of the American Museum of Natural History, 87, 1-100.

Simpson, G. G. 1972. Conspectus of Patagonian Fossil Penguins. American Museum Novitates, 2488, 1-37.

Simpson, G. G. 1981. Notes on some fossil penguins, including a new genus from Patagonia. Ameghiniana, 18, 266- 272.

Stucchi, M. 2002. Una nueva especie de Spheniscus (Aves: Spheniscidae) de la Formación Pisco, Perú. Boletín de la Sociedad Geológica del Perú, 94, 19-26.

Stucchi, M. \& DeVries, T. J. 2003. El registro más antiguo de Sulidae del Perú. Boletín de la Sociedad Geológica del Perú, 96, 117-120.

Stucchi, M., Urbina, M. \& Giraldo, A. 2003. Una nueva especie de Spheniscidae del Mioceno tardío de la Formación Pisco, Perú. Boletín del Instituto Francés de Estudios Andinos, 32, 361-375.

Walsh, S. A. \& Hume, J. P. 2001. A new Neogene marine avian assemblage from North-Central Chile. Journal of Vertebrate Paleontology, 21, 484-491.

Manuscrito recibido: 11 de Diciembre, 2003 Manuscrito aceptado: 19 de Noviembre, 2004 